\title{
Generalized Decoupled Polynomial Chaos for Nonlinear Circuits with Many Random Parameters
}

\author{
Paolo Manfredi, Member, IEEE, Dries Vande Ginste, Senior Member, IEEE, Daniël De Zutter, Fellow, IEEE, \\ Flavio G. Canavero, Fellow, IEEE
}

\begin{abstract}
This letter proposes a general and effective decoupled technique for the stochastic simulation of nonlinear circuits via polynomial chaos. According to the standard framework, stochastic circuit waveforms are still expressed as expansions of orthonormal polynomials. However, by using a point-matching approach instead of the traditional stochastic Galerkin method, a transformation is introduced that renders the polynomial chaos coefficients decoupled and therefore obtainable via repeated non-intrusive simulations and an inverse linear transformation. As discussed throughout the letter, the proposed technique overcomes several limitations of state-of-the-art methods. In particular, the scalability is hugely improved and tens of random parameters can be simultaneously treated within the polynomial chaos framework. Validating application examples are provided that concern the statistical analysis of microwave amplifiers with up to 25 random parameters.
\end{abstract}

Index Terms-Circuit simulation, nonlinear circuits, polynomial chaos, statistical analysis, tolerance analysis, uncertainty.

\section{INTRODUCTION}

With the increasing impact of manufacturing variability on circuit designs, strong attention has been devoted to the development of efficient techniques for statistical circuit analysis. Virtually all circuit simulators rely on the blind and brute-force Monte Carlo (MC) method to collect statistical information, but its computational time rapidly becomes prohibitive as the required number of simulations is typically on the order of many thousands. In recent years, alternative approaches were proposed by exploring the theoretical framework of the generalized polynomial chaos (PC) [1]. According to PC, stochastic variables (voltages and currents in the case of circuit-level simulations) are expanded into series of orthonormal polynomials, whose coefficients readily provide statistical information.

Different strategies are available to solve for the unknown coefficients. Many approaches manipulate the governing equations: recent contributions in the field of circuit simulation include the extension of the stochastic Galerkin method (SGM) to nonlinear circuits [2], [3] and the stochastic testing (ST) method [4]. Unfortunately, the SGM requires the solution

Manuscript received April 16, 2015; accepted May 20, 2015. This work was funded by the Research Foundation Flanders (FWO-Vlaanderen). P. Manfredi is currently an FWO Postdoctoral Research Fellow.

P. Manfredi, D. Vande Ginste and D. De Zutter are with the Electromagnetics Group, Department of Information Technology, Ghent University/iMinds, 9000 Gent, Belgium (e-mail: paolo.manfredi@ugent.be).

F. Canavero is with the EMC Group, Department of Electronics and Telecommunications, Politecnico di Torino, 10129 Torino, Italy. of coupled equations of an augmented size, which dramatically reduces the efficiency when the number of random variables (RVs) is large. Furthermore, although potentially providing optimal accuracy and allowing for efficient adaptive time stepping, the techniques in [2], [4] are intrusive and require the development of an ad hoc simulator.

A second class of methods rely on smart samplingbased strategies, like pseudo-spectral [5] or linear regressionbased [6] techniques. The responses are sampled at suitable values of the RVs and the PC coefficients are retrieved via a weighted summation (quadrature) or interpolation of these samples. These approaches are intrinsically non-intrusive and therefore compatible with standard commercial circuit simulators, but the required number of samples rapidly grows with the number of RVs, even when sparse grids are adopted.

Finally, another recent approach decouples the Galerkinbased equations by exploiting the structure of the resulting augmented matrices [7]. It has the advantage of allowing a faster iterative simulation. Nevertheless, the technique relies on an approximate matrix factorization that applies to Gaussian variability only. Moreover, it requires larger sets of polynomials compared to traditional PC implementations, thus [7] is still limited to a small number of RVs.

Hence, the available techniques suffer from several limitations that prevent their application to complex circuits with many RVs, unless a hierarchy exists among them [8]. The present letter aims to overcome the aforementioned issues by putting forward an alternative and general strategy, leveraging a non-intrusive reformulation of the ST technique [4] by means of a linear transformation that decouples the classical PC coefficients of the circuit variables. Consequently, these coefficients are readily obtained via iterative sampling outside the deterministic solver, thus making the method much easier to implement. Moreover, compared to state-of-the-art collocation techniques, the proposed solution requires fewer samples, thus allowing to account for a much larger number of RVs, while still largely outperforming traditional MC analysis.

\section{Generalized Decoupled Polynomial Chaos}

As most of the circuit simulators are based on the modified nodal analysis representation, we also cast the equations describing a circuit with stochastic parameters in this form:

$$
\mathbf{C}(\boldsymbol{\xi}) \frac{d}{d t} \mathbf{x}(t, \boldsymbol{\xi})+\mathbf{G}(\boldsymbol{\xi}) \mathbf{x}(t, \boldsymbol{\xi})+\mathbf{f}(\mathbf{x}(t, \boldsymbol{\xi}), \boldsymbol{\xi})=\mathbf{u}(t),
$$

where $\boldsymbol{\xi}=\left(\xi_{1}, \ldots, \xi_{d}\right)$ is a $d$-dimensional variable collecting all the (standardized) random circuit parameters; $\mathbf{x}(t, \boldsymbol{\xi}) \in$ 
$\mathbb{R}^{N \times 1}$ collects the node voltages and the currents flowing into the circuit components; $\mathbf{C}(\boldsymbol{\xi}), \mathbf{G}(\boldsymbol{\xi}) \in \mathbb{R}^{N \times N}$ account for the linear memory and memoryless elements, respectively; $\mathbf{f}(\mathbf{x}(t), \boldsymbol{\xi}), \mathbf{u}(t) \in \mathbb{R}^{N \times 1}$ comprise the nonlinear currents and the independent stimuli, respectively. The quantities $\mathbf{C}$, $\mathbf{G}$ and $\mathbf{f}$ are $\boldsymbol{\xi}$-dependent because they are affected by the random circuit parameters. In turn, the waveforms $\mathbf{x}$ are, besides time-dependent, also $\boldsymbol{\xi}$-dependent and, as a result of this randomness, they must be assessed from a statistical standpoint.

According to the general PC framework, the unknown stochastic waveforms are approximated by expansions of multivariate polynomials in the RVs:

$$
\mathbf{x}(t, \boldsymbol{\xi})=\sum_{k=1}^{K} \mathbf{x}_{k}(t) \varphi_{k}(\boldsymbol{\xi})
$$

where the polynomials $\varphi_{k}$ are orthonormal with respect to the probability density function of the random parameters [1]. A total degree truncation is typically adopted, leading to an overall number of $K=(p+d) ! /(p ! d !)$ expansion terms, where $p$ is the maximum total degree of the multivariate polynomials. For circuit simulations, $p=2$ usually provides satisfactory accuracy [3]. From (2), any statistical information is readily derived once the coefficients $\mathbf{x}_{k}(t)$ are known.

To derive the proposed technique, the PC expansion (2) is substituted into (1), yielding

$$
\begin{array}{r}
\mathbf{C}(\boldsymbol{\xi}) \sum_{k=1}^{K} \frac{d}{d t} \mathbf{x}_{k}(t) \varphi_{k}(\boldsymbol{\xi})+\mathbf{G}(\boldsymbol{\xi}) \sum_{k=1}^{K} \mathbf{x}_{k}(t) \varphi_{k}(\boldsymbol{\xi})+ \\
+\mathbf{f}\left(\sum_{k=1}^{K} \mathbf{x}_{k}(t) \varphi_{k}(\boldsymbol{\xi}), \boldsymbol{\xi}\right)=\mathbf{u}(t) .
\end{array}
$$

Next, (3) is enforced to hold at a specific value $\boldsymbol{\xi}=\boldsymbol{\xi}_{m}$ of the random parameters [4], as follows:

$$
\begin{gathered}
\mathbf{C}_{m} \sum_{k=1}^{K} \frac{d}{d t} \mathbf{x}_{k}(t) a_{m k}+\mathbf{G}_{m} \sum_{k=1}^{K} \mathbf{x}_{k}(t) a_{m k}+ \\
\mathbf{f}_{m}\left(\sum_{k=1}^{K} \mathbf{x}_{k}(t) a_{m k}\right)=\mathbf{u}(t),
\end{gathered}
$$

where the notation $a_{m k}=\varphi_{k}\left(\boldsymbol{\xi}_{m}\right)$ has been introduced and the subscript $m$ indicates that the corresponding quantity is evaluated at $\boldsymbol{\xi}_{m}$. So far, all $K$ PC coefficients $\mathbf{x}_{k}(t)$ simultaneously appear in (4) and they are coupled with one another. Yet, in (4), it is possible to introduce the change of variable

$$
\mathbf{y}_{m}(t) \triangleq \sum_{k=1}^{K} \mathbf{x}_{k}(t) a_{m k},
$$

leading to

$$
\mathbf{C}_{m} \frac{d}{d t} \mathbf{y}_{m}(t)+\mathbf{G}_{m} \mathbf{y}_{m}(t)+\mathbf{f}_{m}\left(\mathbf{y}_{m}(t)\right)=\mathbf{u}(t) .
$$

The system (6) is formally identical to (1), but it contains merely deterministic parameters and the transformed variable $\mathbf{y}_{m}(t)$ instead of $\mathbf{x}(t)$. Hence, $\mathbf{y}_{m}(t)$ is the circuit response for the value $\boldsymbol{\xi}_{m}$ of the RVs. By iteratively simulating the circuit for $K$ different samples, a set of $K$ independent waveforms $\mathbf{y}_{m}(t), m=1, \ldots, K$, is obtained. According to the definition (5), these waveforms are now related to the classical PC coefficients $\mathbf{x}_{k}(t)$ by

$$
\underbrace{\left[\begin{array}{c}
\mathbf{y}_{1}(t) \\
\vdots \\
\mathbf{y}_{K}(t)
\end{array}\right]}_{\tilde{\mathbf{y}}(t)}=(\underbrace{\left[\begin{array}{ccc}
a_{11} & \ldots & a_{1 K} \\
\vdots & \ddots & \vdots \\
a_{K 1} & \ldots & a_{K K}
\end{array}\right]}_{\mathbf{A}} \otimes \mathbf{I}_{N}) \underbrace{\left[\begin{array}{c}
\mathbf{x}_{1}(t) \\
\vdots \\
\mathbf{x}_{K}(t)
\end{array}\right]}_{\tilde{\mathbf{x}}(t)},
$$

with $\mathbf{I}_{N}$ the $N \times N$ identity matrix and $\otimes$ the tensor product.

Equation (7) establishes a relationship between the uncoupled waveforms and the classical PC coefficients in terms of a linear transformation. Hence, the following two-step procedure is put forward: i) the stochastic circuit is repeatedly simulated for the $K$ collocation points; ii) the classical PC coefficients are retrieved via the inverse transformation

$$
\tilde{\mathbf{x}}(t)=\left(\mathbf{A}^{-1} \otimes \mathbf{I}_{N}\right) \tilde{\mathbf{y}}(t)
$$

The ST algorithm [4] represents an ideal strategy for the selection of the collocation points, as it applies to any distribution type and yields a non-singular transformation matrix $\mathbf{A}$. The points are a subset of $d$-dimensional Gauss quadrature nodes and a pruning algorithm avoids dealing with initial sets of points of intractable sizes when $d$ is large.

It is important to stress that the outlined method, as opposed to [4], is non-intrusive and therefore much easier to implement. Moreover, other available approaches require the solution of equations that are either larger [5]-[7] or coupled [2], [3]. By way of example, for the common choice $p=2$, the number of simulations $K$ is 66,231 and 496 for 10,20 and $30 \mathrm{RV}$ s respectively, i.e., far less than required by traditional $\mathrm{PC}$ techniques and, of course, by a MC analysis.

\section{ApPlication Examples AND VALIDATION}
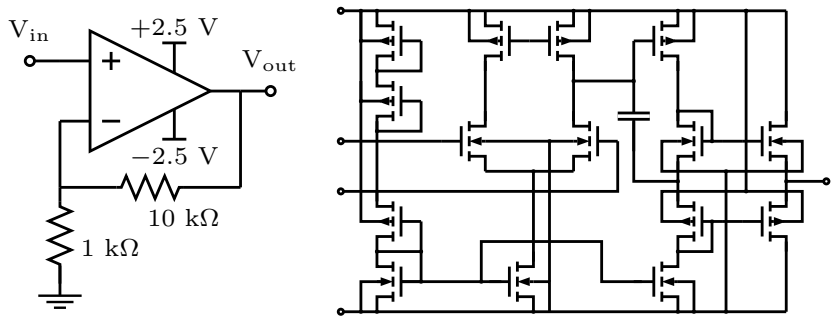

Fig. 1. Non-inverting amplifier of the first application example (left) and internal schematic of its CMOS operational amplifier (right).

The first application example refers to the non-inverting amplifier of Fig. 1(a), where the operational amplifier has the schematic of Fig. 1(b) [9]. In this example, $d=17$ parameters are considered to be random, namely the widths of the fifteen transistors plus the two threshold voltages of the p- and n-type MOS transistors. These parameters are uniformly varied in a range of $\pm 10 \%$ around their nominal values.

Fig. 2 shows the average and standard deviation of the transient output voltage $\mathrm{V}_{\text {out }}(t)$ for an input step signal with an amplitude of $0.01 \mathrm{~V}$ and a risetime of $1 \mathrm{~ns}$, computed with HSPICE via the proposed decoupled PC (dashed and solid lines, respectively) and the MC method (crosses and stars). No appreciable difference is found between the two results. 


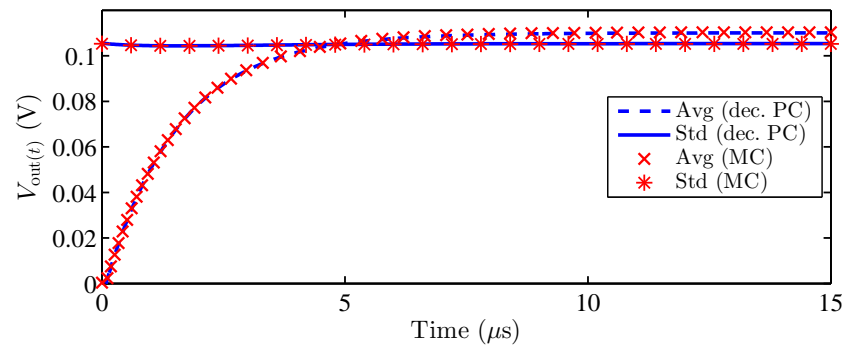

Fig. 2. Average and standard deviation of the amplifier transient output voltage computed with the decoupled (dec.) PC (lines) and MC (markers).

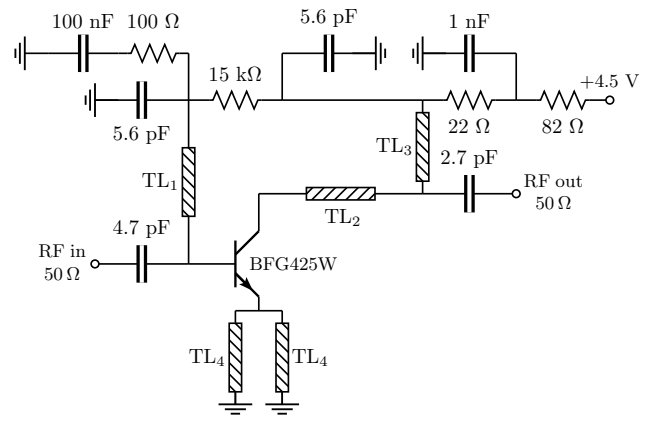

Fig. 3. 2-GHz BJT low-noise amplifier of the second application example.

The second example considers the 2-GHz low-noise amplifier (LNA) of Fig. 3 [10]. For this application, $d=25$ Gaussian $\mathrm{RV}$ s are considered, including the parasitic resistances, capacitances and inductances of the BJT, its forward current gain, and all the lumped components in the amplifier schematic, each with a $10 \%$ relative standard deviation. Moreover, the widths of the four transmission lines are also considered as Gaussian random parameters with a standard deviation of $5 \%$.
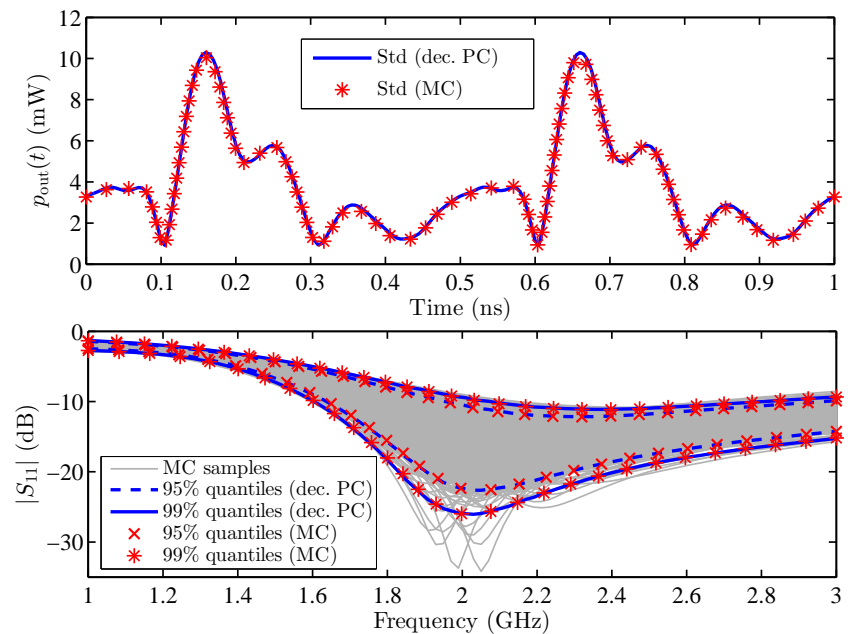

Fig. 4. Top panel: standard deviation of the LNA steady-state output power for a $10 \mathrm{dBm}$ input, computed with both decoupled PC (solid line) and $\mathrm{MC}$ (stars). Bottom panel: variability of $\left|S_{11}\right|$. Thin lines: samples from MC analysis; dashed and solid thick lines: $95 \%$ and $99 \%$ quantiles estimated with the decoupled PC; markers: same quantiles obtained with MC.

The top panel of Fig. 4 shows the standard deviation of the steady-state output power for an input power of $10 \mathrm{dBm}$, computed with the decoupled PC (full line) and MC (stars) via a harmonic balance simulation in HSPICE. The bottom panel provides a statistical assessment of the magnitude of $S_{11}$ due to the variability of the parameters. The thin lines are a superposition of $1000 \mathrm{MC}$ samples from a small-signal (ac) analysis. The dashed and solid thick lines (crosses and stars) are the quantiles bounding $95 \%$ and $99 \%$ of these responses, respectively, computed from the $\mathrm{PC}$ expansions (MC samples).

It is found that, for each case, up to $100000 \mathrm{MC}$ runs are necessary to reach the same accuracy as with the newly proposed technique, thus implying simulation times of several hours. For the two application examples, the decoupled PC instead requires 171 and 351 simulations only!

\section{CONCLUSIONS}

A generalized and effective technique for the decoupled PC-based simulation of nonlinear circuits is outlined in this letter. The method is based on the point matching of the PC-expanded circuit equations and results in a linear transformation of the PC coefficients into uncoupled waveforms. These waveforms are obtained by a limited set of simulations at the collocation points, and the classical PC coefficients are retrieved via a quick inverse linear transformation. The proposed strategy is non-intrusive and therefore compatible with any standard circuit simulator. It allows to overcome several longstanding issues such as coupling in Galerkin equations and sample size in collocation approaches. Excellent accuracy is obtained with a considerably lower number of simulations with respect to the standard MC analysis, even when dozens of RVs are considered.

\section{REFERENCES}

[1] D. Xiu and G. E. Karniadakis, "The Wiener-Askey polynomial chaos for stochastic differential equations," SIAM J. Scientific Computation, vol. 24, no. 2, pp. 619-644, 2002.

[2] M. R. Rufuie, E. Gad, M. Nakhla, and R. Achar, "Generalized Hermite polynomial chaos for variability analysis of macromodels embedded in nonlinear circuits," IEEE Trans. Compon. Packag. Manuf. Technol., vol. 4, no. 4, pp. 673-684, Apr. 2014.

[3] P. Manfredi, D. Vande Ginste, D. De Zutter, and F. G. Canavero, "Stochastic modeling of nonlinear circuits via SPICE-compatible spectral equivalents," IEEE Trans. Circuits Syst. I, Reg. Papers, vol. 61, no. 7, pp. 2057-2065, Jul. 2014.

[4] Z. Zhang, T. A. El-Moselhy, I. M. Elfadel, and L. Daniel, "Stochastic testing method for transistor-level uncertainty quantification based on generalized polynomial chaos," IEEE Trans. Comput.-Aided Des. Integr. Circuits Syst., vol. 32, no. 10, pp. 1533-1545, Oct. 2013.

[5] A. Rong and A. C. Cangellaris, "Interconnect transient simulation in the presence of layout and routing uncertainty," in Proc. IEEE 20th Conf. Elect. Performance Electron. Packaging Syst., San Jose, CA, Oct. 2011, pp. $157-160$.

[6] D. Spina, F. Ferranti, T. Dhaene, L. Knockaert, G. Antonini, and D. Vande Ginste, "Variability analysis of multiport systems via polynomial-chaos expansion," IEEE Trans. Microw. Theory Tech., vol. 60, no. 8, pp. 23292338, Aug. 2012.

[7] M. R. Rufuie, E. Gad, M. Nakhla, R. Achar, and M. Farhan, "Fast variability analysis of general nonlinear circuits using decoupled polynomial chaos," in Proc. IEEE 18th Workshop on Signal and Power Integrity, Ghent, Belgium, May 2014, pp. 1-4.

[8] Z. Zhang, X. Yang, I. V. Oseledets, G. E. Karniadakis, and L. Daniel, "Enabling high-dimensional hierarchical uncertainty quantification by ANOVA and tensor-train decomposition," IEEE Trans. Comput.-Aided Des. Integr. Circuits Syst., vol. 34, no. 1, pp. 63-76, Jan. 2015.

[9] [Online]. Available: https://awrcorp.com/download/faq/english/examples/ 3_StageCMOS_Opamp.aspx

[10] T. Buss, "2GHz low noise amplifier with the BFG425W," Philips Semiconductors B.V., Nijmegen, The Netherlands, Application Note RNRT45-96-B-773, Nov. 1996. 